\section{ARTACUS: AN OPEN-LABEL, MULTICENTER, PHASE 1B/2 STUDY OF RP1 IN SOLID ORGAN TRANSPLANT RECIPIENTS WITH ADVANCED CUTANEOUS MALIGNANCIES}

${ }^{1}$ Jason Luke*, ${ }^{2}$ Michael Migden, ${ }^{3}$ Wanxing Chai-Ho, ${ }^{4}$ Diana Bolotin, ${ }^{5}$ Trisha Wise-Draper, ${ }^{6}$ Andrew Poklepovic, ${ }^{7}$ Douglas Laux, ${ }^{8}$ Meenal Kheterpal, ${ }^{9}$ Claire Verschraegen, ${ }^{10}$ Frances Collichio, ${ }^{11}$ Jose Lutzky, ${ }^{12}$ Gregory Daniels, ${ }^{13}$ Katy Tsai, ${ }^{14}$ Susan Navia, ${ }^{14}$ Henry Castro, ${ }^{14}$ Praveen Bommareddy, ${ }^{14}$ Andrea Pirzkall, ${ }^{14}$ Robert Coffin. ${ }^{1}$ UPMC Hillman Cancer Center, Pittsburgh, PA, USA; ${ }^{2}$ University of Texas MD Anderson Cancer C, Houston, TX, USA; ${ }^{3}$ UCLA David Geffen School of Medicine, Los Angeles, USA; ${ }^{4}$ University of Chicago, Chicago, IL, USA; ${ }^{5}$ UC Vontz Center for Molecular Studies, Cincinnati, OH, USA; ${ }^{6}$ Virginia Commonwealth University, Richmond, MA, USA; ${ }^{7}$ University of lowa Hospitals and Clinics, lowa City, IA, USA; ${ }^{8}$ Duke University, Durham, NC, USA; ${ }^{9}$ Ohio State University Comprehensive Ctr, Columbus, OH, USA; ${ }^{10}$ UNC, Chapel Hill, Chapel Hill, NC, USA; ${ }^{11}$ Sylvester Comprehensive Cancer Center, Miami, FL, USA; ${ }^{12}$ UC San Diego, La Jolla, CA, USA; ${ }^{13}$ University of California San Francisco, San Francisco, CA, USA; ${ }^{14}$ Replimune, Woburn, MA, USA

Background Solid organ transplantation (SOT) has emerged as an important lifesaving procedure for patients with a wide range of end-organ diseases characterized by dysfunction or specific organ function failure. SOT rejection is a major complication requiring patients (pts) to undergo lifelong immunosuppression to prevent allograft rejection. ${ }^{1}$ Skin cancers (SCs) including cutaneous squamous cell carcinoma (CSCC) are common post transplant malignancies. ${ }^{2} \mathrm{SC}$ in SOT pts is generally managed with surgical resection, radiation therapy and chemotherapy or targeted therapy. Use of immune checkpoint inhibitors in SOT recipients has improved outcomes but are associated with the high risk of allograft rejection. ${ }^{3-5}$ Thus, there is a high unmet need for a safe and effective treatment that also protects pts from allograft rejection. RP1 is an oncolytic virus (HSV-1) that expresses a fusogenic glycoprotein (GALV-GP R-) and granulocyte macrophage colony stimulating factor (GM-CSF). In preclinical studies, RP1 induced immunogenic tumor cell death and provided potent systemic antitumor activity ${ }^{6}$ and clinical data in combination with nivolumab has demonstrated a high rate of deep and durable response in patients with advanced SCs. ${ }^{7}$ The objective of this study is to assess the safety and efficacy of single agent RP1 in kidney and liver transplant recipients with SCs, with focus on CSCC. After determining the safety and tolerability in the initial cohort with kidney and liver transplants the study may also enroll heart and lung transplant recipients.

Methods This study will enroll up to 65 evaluable allograft transplantation pts with locally advanced or metastatic SCs. Key inclusion criteria are pts with confirmed recurrent, locally advanced or metastatic CSCC and up to 10 pts with nonCSCC SC, stable allograft function and ECOG performance status of $\leq 1$. Pts with prior systemic anti-cancer treatment are allowed. Key exclusion criteria are prior treatment with an oncolytic therapy, active herpetic infections or prior complications of HSV-1 infection and a history of organ graft rejection within 12 months. Pts will receive an initial dose of $1 \mathrm{x}$ $10^{\wedge} 6$ plaque-forming units (PFU) of RP1. Two weeks later they will receive $1 \times 10^{\wedge} 7$ PFU of RP1 and continue every two weeks until pre-specified study endpoints are met. RP1 will be administered by intra-tumoral injection including through imaging guidance as clinically appropriate. The primary objective of the trial is to assess efficacy determined by ORR and safety of single agent RP1. Additional secondary endpoints include DOR, CR, DCR, PFS and OS.

Trial Registration NCT04349436

\section{REFERENCES}

1. Frohn C, Fricke L, Puchta JC, Kirchner H. The effect of HLA-C matching on acute renal transplant rejection. Nephrol Dial Transplant 2001:16(2):355-60.

2. Madeleine MM, Patel NS, Plasmeijer El, Engels EA, Bouwes Bavinck JN, Toland $A E$, Green $A C$; the Keratinocyte Carcinoma Consortium (KeraCon) Immunosuppression Working Group. Epidemiology of keratinocyte carcinomas after organ transplantation. Br J Dermatol 2017;177(5):1208-1216.

3. Spain L, Higgins R, Gopalakrishnan K, Turajlic S, Gore M, Larkin J. Acute renal allograft rejection after immune checkpoint inhibitor therapy for metastatic melanoma. Ann Oncol 2016;27(6):1135-1137.

4. Herz S, Höfer T, Papapanagiotou M, Leyh JC, Meyenburg S, Schadendorf D, Ugurel S, Roesch A, Livingstone E, Schilling B, Franklin C. Checkpoint inhibitors in chronic kidney failure and an organ transplant recipient. Eur I Cancer 2016;67:66-72

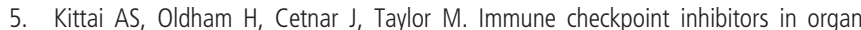
transplant ptss. J Immunother 2017;40(7):277-281.

6. Thomas S, Kuncheria L, Roulstone V, Kyula JN, Mansfield D, Bommareddy PK, Smith H, Kaufman HL, Harrington KJ, Coffin RS. Development of a new fusionenhanced oncolytic immunotherapy platform based on herpes simplex virus type 1. J Immunother Cancer 2019 10;7(1):214.

7. Middleton M, Aroldi F, Sacco J, Milhem M, Curti B, Vanderwalde A, Baum S, Samson A, Pavlick A, Chesney J, Niu J, Rhodes T, Bowles T, Conry R, OlssonBrown A, Earl-Laux D, Kaufman H, Bommareddy P, Deterding A, Samakoglu S, Coffin R, Harrington K. 422 An open-label, multicenter, phase 1/2 clinical trial of RP1, an enhanced potency oncolytic HSV, combined with nivolumab: updated results from the skin cancer cohorts. I Immunother Cancer 2020;8(3): doi: 10.1136/jitc-2020-SITC2020.0422

Ethics Approval The study was approved by institutional review board or the local ethics committee at each participating site. Informed consent was obtained from patients before participating in the trial.

http://dx.doi.org/10.1136/jitc-2021-SITC2021.550 